\title{
REVISÃO SOBRE O USO DE SENSORIAMENTO REMOTO NA DETECÇÃO DE VAZAMENTOS DE ÓLEO NO MAR
}

\author{
Nájla Vilar Aires de Moura \\ Doutoranda do Programa de Pós-Graduação em Geografia, Universidade de Brasília, \\ Brasília, DF, Brasil \\ najlavilar@hotmail.com \\ Osmar Abílio de Carvalho Júnior \\ Professor Dr. do Departamento de Geografia, Universidade de Brasília \\ Brasília, DF, Brasil \\ osmarjr@unb.br \\ Roberto Arnaldo Trancoso Gomes \\ Professor Dr. do Departamento de Geografia, Universidade de Brasília \\ Brasília, DF, Brasil \\ robertogomes@unb.br \\ Renato Fontes Guimarães \\ Professor Dr. do Departamento de Geografia, Universidade de Brasília \\ Brasília, DF, Brasil \\ renatofg@unb.br
}

\begin{abstract}
RESUMO
Vazamentos de óleo no mar são de grande preocupação pública por representarem riscos para o meio ambiente e para a população costeira. Com o crescimento da exploração do petróleo e da movimentação de navios aumenta a possibilidade de acidentes, nesse contexto o sensoriamento remoto é usado como ferramenta indispensável para a detecção e monitoramento das manchas de óleo na superfície marítima. $O$ presente trabalho traz uma revisão das pesquisas recentes desenvolvidas na área, que sobretudo visam melhorar a acurácia da detecção e classificação das manchas com potencial de serem derramamento de óleo. O Radar de Abertura Sintética (SAR) destaca-se como o sensor mais usado, por ser eficiente mesmo com a presença de nuvens e no período noturno. O desafio atual é a necessidade de criar metodologias eficientes na distinção de manchas de óleo de outras feições escuras que aparecem na imagem de radar formadas por fenômenos diversos que podem gerar falsos-positivo.
\end{abstract}

Palavras-chave: Radar. Classificação. Monitoramento.

\section{REVIEW OF REMOTE SENSE USE FOR OIL SPILL DETECTION IN THE SEA}

\begin{abstract}
Oil spills at sea are a major public concern as they represent risks to environment and coastal population. The growth of oil exploration and ship movement increases the possibility of accidents, in this context remote sensing is used as an indispensable tool for the detection and monitoring of oil slicks on the sea surface. The present work presents a review of the recent research developed in the area, which mainly aims to improve the accuracy of detection and classification of oil spill. Synthetic Aperture Radar (SAR) stands out as the most used sensor, being efficient even in the presence of clouds and at night. The current challenge is the need to create efficient methodologies in distinguishing oil slicks from other dark features that appear in the radar image formed by various phenomena that can generate lookalikes.
\end{abstract}

Keywords: Radar. Classification. Monitoring.

\section{INTRODUÇÃO}

Anualmente milhares de toneladas de óleo são derramadas nos oceanos, seja por causas antropogênicas, seja por causas naturais. Os derramamentos provocados pela ação humana podem ser intencionais ou acidentais, envolvendo tanques de embarcações, ruptura de dutos e mal

$\begin{array}{lllll}\text { Caminhos de Geografia } & \text { Uberlândia-MG } & \text { v. 21, n. } 75 & \text { Jun/2020 } & \text { p. 214-224 Página } 214\end{array}$


funcionamento de plataformas de exploração de petróleo. Os vazamentos de causas naturais têm origem em rupturas ocorridas no fundo marinho (BREKKE e SOLBERG, 2005b; PISANO et al., 2016).

Com o aumento da exploração comercial do petróleo e da movimentação de navios é crescente o registro de ocorrência de vazamentos (YU, 2007), especialmente aqueles oriundos de descargas ilegais e intencionais de, por exemplo, água oleosa oriunda das lavagens de tanques, porões e conveses de embarcações e da liberação de água de produção de plataformas de petróleo contendo teor de óleo acima da especificação legal (MISRA e BALAJI, 2017). Adicionalmente, a identificação, prevenção e atendimento a emergências envolvendo vazamentos de óleo ocorridos no mar, são de preocupação pública uma vez que apresentam grande potencial de danos letais aos ecossistemas marítimos e costeiros (ESPEDAL e JOHANNESSEN, 2000; FISCELLA et al., 2000; SINGHA et al., 2013; FUSTES et al., 2014; ZHANG et al., 2014; ABASCAL et al., 2017; ALPERS et al., 2017). Ressalta-se que no presente trabalho é usada a palavra "óleo" para qualquer forma de hidrocarboneto (petróleo e seus derivados) oriundo da exploração de petróleo ou da movimentação de navios, provocados intencionalmente ou por acidentes, conforme conceituação da Lei $\mathrm{n}^{0}$ 9.966/2000 (BRASIL, 2019).

Portanto, torna-se imprescindível a existência de ferramentas que contribuam para a fiscalização dos vazamentos irregulares e para assegurar o cumprimento das regulamentações nacionais e internacionais para o controle das atividades e proteção ambiental (ESPEDAL e JOHANNESSEN, 2000; SINGHA et al., 2013). Para o atendimento eficiente a um acidente dessa natureza é essencial que as ações de resposta sejam adotadas de maneira rápida e que a detecção ocorra o mais cedo possível (KERAMITSOGLOU et al., 2006). Nesse contexto, o sensoriamento remoto é de grande importância para a proteção ambiental das áreas costeiras e primordial para a vigilância marítima uma vez que propicia a identificação precoce de acidentes envolvendo o vazamento de óleo, além de ser capaz de identificar eventos em áreas remotas e de difícil acesso (SINGHA et al., 2013; ZHANG et al., 2014). Fingas e Brown $(2014 ; 2018)$ em revisão sobre o assunto, indicam o uso do sensoriamento remoto como ferramenta eficiente na gestão ambiental de um vazamento de óleo em diferentes etapas: mapeamento dos vazamentos, vigilância e detecção, produção de provas para processos judiciais, fiscalização do cumprimento de leis sobre descarga de navios, orientação para tomada de medidas de controle do vazamento e determinação da trajetória da mancha de óleo.

Os principais métodos de sensoriamento remoto para a deteç̧ão de vazamentos de óleo estão baseados no uso de sensores óticos e de radar, sendo que o SAR (Synthetic Aperture Radar) possui maior utilização devido a sua capacidade de funcionamento sob qualquer condição atmosférica e de luminosidade, incluindo durante a noite (PISANO, et al.; 2016). Segundo Garcia-Pineda et al. (2013), o uso de radar apresenta maiores vantagens para o monitoramento de vazamento de óleo nos oceanos, uma vez que é um sistema ativo, ao contrário dos sensores óticos que precisam de iluminação solar e são limitados quando há a presença de nuvens ou baixa luminosidade.

O presente estudo tem por objetivo apresentar as metodologias de sensoriamento remoto recentemente desenvolvidas e aplicadas no mundo para a detecção de óleo na superfície do mar, com destaque para aquelas que utilizam imagens SAR, uma vez que estas são amplamente usadas para este fim e mostram ser mais eficientes (GARCIA-PINEDA et al., 2013).

\section{SENSORES USADOS PARA DETECTAR MANCHAS DE ÓLEO E SUAS CARACTERÍSTICAS}

Atualmente diversas técnicas de sensoriamento remoto são usadas para a detecção dos vazamentos de óleo no mar, especialmente por permitirem o monitoramento de áreas grandes em relativo curto espaço de tempo, em contraste com o monitoramento visual realizado por aeronaves e embarcações que têm elevados custos e área de abrangência limitada. Os sensores mais utilizados são Side Looking Airborne Radar (SLAR), Infravermelho (IR), Ultravioleta (UV), laser fluorensores (presentes sobretudo em aeronaves) e, Synthetic Aperture Radar (SAR) e sensores óticos, presentes em diversas plataformas de satélites e aeronaves (ESPEDAL e JOHANNESSEN, 2000; FINGAS e BROWN, 2014).

Fingas e Brown $(2014 ; 2018)$ apresentam as propriedades óticas do óleo e suas características específicas de comportamento no uso de diversos sensores. Caracterizam os sensores visíveis, infravermelho, NIR, ultravioleta, laser fluorosensores, micro-ondas (passivos e radares), apresentando para este o detalhamento mínimo do processamento. Considerando o uso de sensores que operam na faixa do visível, os autores afirmam que o óleo possui reflectância maior que a água, mas sem 
apresentar características específicas quanto a absorção e reflexão, não existindo, portanto, meios de identificar positivamente o óleo da água com esses sensores. Especificamente sobre os sensores presentes em satélites, os autores afirmam que existe a desvantagem de serem imagens obtidas com intervalos de tempo longos e que demandam longo tempo de processamento, além de serem limitados quando há a presença de nuvens e sofrerem interferência do reflexo do sol em determinadas partes das imagens. Brekke e Solberg (2005b) também afirmam que as condições atmosféricas são limitadoras para o uso de sensores que operam na faixa do visível.

Sensores que operam na faixa do infravermelho baseiam-se na diferença de temperatura entre a água e o óleo. O óleo absorve parte da radiação solar e reemite parte dela como energia termal, dessa maneira, as manchas de óleo grossas aparecem nas imagens como quente, as de espessura intermediária aparecem mais frias, enquanto as manchas finas não serão detectadas. No entanto, durante a noite camadas grossas de óleo podem ser detectadas como frias uma vez que esfriam mais rápido que a água ao redor. Esses sensores possuem limitações, principalmente quando o óleo forma emulsão com a água (BREKKE e SOLBERG, 2005b; FINGAS e BROWN, 2014).

Os sensores ultravioletas têm o seu funcionamento decorrente da elevada reflexividade do óleo nessa faixa. Esses instrumentos são capazes de detectar óleo até em finas camadas, contudo, têm como limitação o fato de não poderem ser usados a noite e por gerar falso-positivos em manchas causas pelo vento, por reflexos do sol e por material biogênico, não sendo uma ferramenta muito utilizada (BREKKE e SOLBERG, 2005b; FINGAS e BROWN, 2014).

Outro tipo de sensor remoto usado para identificar a presença de óleo na água faz uso de laser fluorosensores. São sensores que se baseiam no fato de certos componentes aromáticos do óleo se tornarem fluorescentes, sobretudo ao receberem luzes ultravioletas. São capazes de detectar o óleo presente na superfície da água e também contido na coluna d'água até 2 metros de profundidade. Esses sensores são indicados para a detecção do óleo uma vez que a fluorescência do óleo apresenta comprimento de onda bastante específico a ponto de não ser confundido com outras substâncias presentes na água (FINGAS e BROWN, 2014).

Sensores hiperespectrais têm potencial para identificação detalhada de materiais e melhor estimativa de sua abundância, uma vez que permite o detalhamento físico-químico da substância presente na superfície imageada. Seus diferentes comprimentos de onda podem ser usados para reconhecimento da assinatura espectral de diferentes tipos de óleo, também pode ser usado para distinção de manchas formadas por outros materiais que tenham aspecto similar (BREKKE e SOLBERG, 2005b).

Sensores que operam na faixa das micro-ondas são aqueles mais comumente usados para a detecção de poluição de óleo, principalmente sensores ativos como os radares. São preferíveis, comparados aos sensores óticos, por serem eficientes em quaisquer condições de tempo e luminosidade. Os mais comuns são o SLAR (Side-Looking Airborne Radar) que tem como desvantagem ser caro e estar presente em aeronaves que têm área de cobertura limitada, e o Synthetic Aperture Radar (SAR). O uso de SAR tem a vantagem de permitir o monitoramento em qualquer condição de luminosidade e climática (ESPEDAL e JOHANNESSEN, 2000; BREKKE e SOLBERG, 2005b; FINGAS e BROWN, 2014).

Sensores MWR (microwave radiometer) são sensores passivos que captam as micro-ondas refletidas pela superfície, oriundas da radiação do espaço. São usados com maior frequência para medição da espessura da mancha de óleo e cálculo do volume vazado (BREKKE e SOLBERG, 2005b; FINGAS e BROWN, 2014).

Destaca-se que nenhum dos sensores pode individualmente fornecer todas as informações necessárias para o gerenciamento de um incidente envolvendo vazamento de óleo, cada um apresenta vantagens e desvantagens, tendo aplicações específicas. Sensores presentes em satélites, como alguns SAR, apresentam como vantagem o fato de terem baixos custos, por outro lado têm baixa resolução temporal e espacial, quando comparados aos sensores presentes em aeronaves (GARCIA-PINEDA et al., 2013).

Segundo Brekke e Solberg (2005b), o SAR é o mais eficiente sensor satelital para a detecção de óleo, embora não tenha capacidade para detectar a espessura da mancha e o tipo de óleo. Adicionalmente, afirma que o uso do SAR apresenta como desvantagem o fato de não distinguir manchas formadas por óleo, daquelas formadas por outros de fenômenos que também geram na imagem manchas escuras, podendo gerar falso-positivos. 


\section{CARACTERÍSTICAS DO SAR E SUA APLICAÇÃO NA IDENTIFICAÇÃO DE MANCHAS DE ÓLEO}

O SAR é um sensor ativo que emite micro-ondas em intervalo de frequência que inclui a banda $X$ ( 8 a $12 \mathrm{GHz}, 2,50$ a $4 \mathrm{~cm}$ ), banda C ( 4 a $8 \mathrm{GHz}, 4$ a $8 \mathrm{~cm}$ ) e banda L ( 1 a $2 \mathrm{GHz}, 15$ a $30 \mathrm{~cm}$ ). Por meio das micro-ondas é detectado o alcance, a velocidade e outras características dos objetos remotos (GARCIA-PINEDA et al., 2013). Assim, o SAR emite micro-ondas e grava os sinais refletidos pelos objetos alvos (ZHANG et al., 2014).

Três componentes principais dominam o retorno de energia da superfície da Terra a um satélite equipado com SAR: 1) a geometria entre as posições relativas do satélite e da imagem (ou seja, espectros direcionais de ondas e ângulos de incidência do feixe); 2) rugosidade; e 3) as propriedades dielétricas dos materiais da superfície (GARCIA-PINEDA et al., 2013).

As propriedades de dispersão de um material dependem da polarização do sinal do radar tanto de entrada como de saída, tendo sido demonstrado por alguns estudos que para a identificação dos vazamentos de óleo a configuração mais adequada para o SAR é a VV (propagação vertical da onda na emissão e na transmissão e na recepção do sinal) (BREKKE e SOLBERG, 2005b; FINGAS e BROWN, 2014).

Nos trabalhos de sensoriamento remoto desenvolvidos em áreas marítimas o mecanismo de atuação do SAR ocorre a partir da interação das micro-ondas com as ondas capilares formadas na superfície da água (ZHANG et al., 2014), conforme detalhado no próximo item.

\section{CARACTERÍSTICAS DAS MANCHAS DE ÓLEO E FALSO-POSITIVOS PRESENTES NAS IMAGENS SAR}

Em condições normais, a superfície do mar aparece nas imagens de radar de maneira brilhante, uma vez que as ondas capilares, formadas pela incidência do vento na água, refletem a energia emitida pelo radar. Contudo, a existência de óleo no mar ameniza o efeito dessas ondas, uma vez que torna a superfície mais plana e reduz o coeficiente de retroespalhamento do radar, dessa forma a presença de óleo na água aparece na imagem como manchas escuras que contrastam com a água ao redor sem a presença de óleo (FISCELLA et al., 2000; FINGAS e BROWN, 2014; PISANO et al., 2016; ALPERS et al., 2017).

No entanto, diversas outras causas podem produzir o mesmo fenômeno na superfície da água, gerando, da mesma forma, manchas escuras nas imagens (BREKKE e SOLBERG, 2005b; MARGHANY, 2001; ZHANG et al., 2014). Os denominados falso-positivos (lookalike) podem ocorrer em decorrência de fenômenos naturais, tais como: óleos naturais, zonas de cisalhamento do relevo marítimo, células de chuva, presença de água doce próximas a foz de rios, áreas de calmaria de vento, sombras atrás de porções de terra e de estruturas, camadas de algas abaixo da superfície da água que fazem com que a água acima se torne calma, presença de gelo, substâncias biogênicas (produzidas, por exemplo, por fitoplâncton, espermatozoides de baleias e peixes ou substâncias oriundas de sua decomposição), áreas abrigadas, água quente que muda a estabilidade da interface ar/mar, fluxo divergente associado a ondas internas ou fluxo de maré sobre bancos de areia e água oriunda de tempestade que flui da terra para o mar. Falso-positivos também surgem em decorrência da ação humana como: ocorrência de turbulência na água encontrada após a passagem de navios, vazamentos de água doce de navios, águas residuais oriundas de plantas industriais ou áreas urbanas (sobretudo esgoto), derramamento de óleo durante limpeza de tanque de navios que transportam óleo vegetal ou óleo de peixe oriundo de navios de pesca (ESPEDAL e JOHANNESSEN, 2000; BREKKE e SOLBERG, 2005b; TOPOUZELIS et al., 2007; FINGAS e BROWN, 2014; ZHANG et al., 2014; PISANO et al., 2016; ALPERS et al., 2017).

Dessa forma, considerando o elevado número de fenômenos na superfície marítima que podem gerar o aparecimento de manchas escuras em imagens de radar, é essencial o desenvolvimento de metodologias que diferenciem uma feição da outra, sendo esse o principal desafio a ser superado (FINGAS e BROWN, 2014; ALPERS et al., 2017). Segundo estudo de Fingas e Brown (2014) foram detectados como falso-positivo $20 \%$ das manchas registradas como óleo.

Outro fator limitante para a confirmação de óleo diz respeito à velocidade adequada do vento, havendo variação da faixa ideal entre as pesquisas analisadas: (a) de $3 \mathrm{~m} / \mathrm{s}$ até $8 \mathrm{~m} / \mathrm{s}$ (FINGAS e BROWN, 2014); (b) de $2 \mathrm{~m} / \mathrm{s}$ até $10 \mathrm{~m} / \mathrm{s}$ (FISCELLA et al., 2000); (c) detecção possível entre 2 e 12 $\mathrm{m} / \mathrm{s}$ e intervalo otimizado entre 5 e $6 \mathrm{~m} / \mathrm{s}$ (JONES, 2001). Em condições onde a velocidade do vento é baixa (inferior a $2 \mathrm{~m} / \mathrm{s}$ ), a detecção de óleo através de radar não é possível devido à redução e 
aplainamento das ondas capilares (PISANO et al., 2016). Em contraste, a elevada velocidade do vento intensifica a formação de ondas de maneira que camadas de óleo, mesmo as espessas, não teriam condições de amenizar o efeito de ondas intensas. Adicionalmente, o óleo presente na água submetida a ventos intensos sofre dispersão mecânica, desaparecendo rapidamente da superfície (FISCELLA et al., 2000; BENTZ et al., 2005; ZHANG et al., 2014; PISANO et al., 2016).

Diante das diversas condições que podem gerar falso-positivos é necessário observar durante a análise das imagens, sobretudo nas abordagens manuais, a forma da mancha como fator que contribui para a distinção entre manchas de óleo e outros fenômenos, considerando principalmente as características das bordas, a extensão e a área ao redor. As manchas de óleo tendem a ter as bordas bem definidas e o formato alongado e retilíneo ou levemente curvado (TOPOUZELIS, 2008). Guo e Zhang (2014) e Topouzelis e Singha (2016) sintetizam como o principal problema na detecção de vazamentos de óleo, justamente a necessidade de fazer a discriminação entre os vazamentos reais e os falso-positivos.

\section{PESQUISAS PARA IDENTIFICAÇÃO DE MANCHAS DE ÓLEO EM IMAGENS SAR}

Duas formas de abordagem metodológica são usadas para a detecção de vazamentos de óleo em imagens SAR: a manual e a semiautomática e/ou inteiramente automática. A primeira abordagem é a mais popular, pouco complexa e de fácil reprodução, sendo dependente da experiência do analista em saber distinguir, principalmente através da forma e da análise dos arredores, um vazamento real de um falso-positivo (TOPOUZELIS, 2008; MISRA e BALAJI, 2017). Adicionalmente, o analista deve considerar informações da área da imagem como velocidade do vento, correntes marítimas e períodos de insurgência de algas (TOPOUZELIS, 2008).

A abordagem semiautomática e/ou totalmente automática é considerada mais complexa e difícil de ser reproduzida, demandando conhecimento dos padrões das imagens e das teorias de classificação (TOPOUZELIS, 2008). Para o processamento das imagens SAR, nessa abordagem, são adotadas fases essenciais (TOPOUZELIS, 2008; LANG et al., 2017).

1. Deteç̧ão e isolamento de todas as formações escuras apresentadas na imagem. Esta etapa é resultado do processamento de limiarização e segmentação, visa destacar as feições com potencial de serem vazamentos de óleo.

2. Extração de parâmetros estatísticos das manchas escuras. Onde é gerado um vetor com as características que descrevem quantitativamente as manchas. Estas características estão relacionadas com a geometria da formação (área, perímetro, forma das bordas), o comportamento físico (valor médio de retroespalhamento) e contexto da mancha na imagem (distância de navios, plataformas e linha de costa).

3. Teste dos valores extraídos em relação a valores pré-definidos, que caracterizam os vazamentos de óleo e as manchas formadas por outros fenômenos. Esses valores são geralmente determinados por meio de considerações fenomenológicas e avaliações estatísticas.

4. Classificação das manchas escuras. Nessa etapa ocorre a distinção entre vazamentos de óleo e falso-positivos. Vários classificadores são usados, podendo ser usadas diversas abordagens estatísticas através do cálculo de probabilidades como através de redes neurais, lógica difusa, etc. (TOPOUZELIS, 2008; LANG et al., 2017).

Zhang et al. (2014) estabelecem, de maneira similar, a estrutura padrão dos algoritmos, destacando a importância da etapa de pré-processamento como procedimento fundamental para melhorar a precisão da detecção de óleo. Seguindo essa linha de ação, Espedal e Johannessen (2000) apresentam a metodologia usada pelo Nansen Environmental and Remote Sensing Center (NERSC) da Noruega para distinguir manchas de vazamentos naturais inofensivos, daquelas provocadas pela ação humana, tendo sido desenvolvido algoritmo supervisionado que contém os seguintes passos: processamento inicial das imagens; análise direta (considerando a fonte do vazamento caso esteja visível, forma, tamanho e dimensão, textura, amortecimentos e gradientes); análise contextual (considerando informações sobre ventos, fontes de poluição, chuvas, temperatura/estação, presença de gelo e a topografia do fundo oceânico); modelagem do comportamento da mancha (o modelo usado deve ser baseado em condições ambientais relevantes para o vazamento em estudo); 
estabelecimento de conclusões com base na combinação de resultados. Os autores concluem que o algoritmo fornece um procedimento padrão a ser seguido e garante que os mesmos critérios sejam aplicados cada vez que uma mancha é analisada.

Em trabalho sobre os desafios e dificuldades na classificação de manchas escuras, Alpers et al. (2017) destacam a necessidade de que os algoritmos de detecção de vazamentos de óleo sejam melhorados consideravelmente, incluindo informações sobre: 1) a distribuição de clorofila-a (indicando a presença provável de manchas biogênicas); 2) a localização das plataformas de petróleo e óleo; 3) a localização das correntes marítimas superficiais; 4) informações sobre a condição dos ventos próximos da superfície do mar; 5) a localização dos bancos de areia (que aparecem escuros nas imagens SAR); 6) a localização de frentes (limite entre duas massas de água com características distintas que muitas vezes se manifestam como linhas escuras em imagens SAR); 7) diferença entre a temperatura do ar e do mar; 8) as distribuições de células de chuva (usando imagens de radar meteorológico); e, o mais importante, 9) incluindo informações sobre o tráfego de navio.

Bentz et al. (2005) apresentam metodologia desenvolvida para o monitoramento ambiental de áreas costeiras e oceânicas com múltiplos sensores orbitais e afirmam ser necessário a análise do tamanho, forma, textura e contexto espacial das manchas escuras, provocadas pelo baixo retroespalhamento dos pulsos emitidos, visando a identificação do fenômeno que deu origem. Dessa forma, a metodologia desenvolvida cruza dados de temperatura da superfície da água, direção e velocidade do vento, presença de nuvens, forma da mancha e concentração de clorofila-a, para identificação da origem das manchas na imagem de radar.

Brekke e Solberg (2005a; 2008) propõem metodologia onde a estrutura do algoritmo é composta por: 1) detector de manchas escuras; 2) ferramenta de extração; e 3) classificador das manchas escuras. As autoras sugerem a introdução da regularização das matrizes de covariância para diminuir o número de falso-positivos e comparam o método classificação Support Vector Machines (SVM) com o classificador estatístico regularizado. O estudo propõe procedimento de classificação em dois passos para a detecção de vazamentos de óleo em imagens SAR, consistindo em um classificador estatístico regularizado e uma estimativa de confiança automática das manchas detectadas. Essa combinação leva a um desempenho de classificação aceitável se o algoritmo for executado antes de verificação visual (com envio de aeronave/navio para confirmação do vazamento).

Fiscella et al. (2000) desenvolveram metodologia para distinção de manchas em imagens SAR, a partir do uso de informações estatísticas obtidas de acidentes anteriores com fontes conhecidas, baseada em características físicas e geométricas de manchas tanto de vazamentos quando de feições naturais que podem representar falso-positivos. Para aplicação da metodologia inicialmente foram selecionadas somente imagens com a presença de manchas escuras, na sequência foram recortadas as cenas com as manchas (de forma manual ou automática), em seguida aplicada máscara para descartar as porções de terras e as manchas escuras de baixa relevância (aquelas muito grandes, com maior probabilidade de ser área de ventos, e as muito pequenas, que potencialmente representariam vazamentos insignificantes). Para a elaboração do algoritmo foram quantificadas informações sobre perímetro e área da mancha, média NRCS (Normalized Radar Cross-section) e fator de forma; a etapa posterior foi a classificação usando dois diferentes métodos: Mahalanobis e classificação de probabilidade composta, ambos com resultados semelhantes. No estudo foram usadas 80 amostras de vazamentos e 43 de falso-positivos. O método aplicado foi capaz de classificar $80 \%$ das manchas corretamente e teve resultados similares de classificação feita por interpretação visual de imagens do mesmo grupo de manchas.

Garcia-Pineda et al. (2009) desenvolveram um algoritmo usando rede neural baseado na textura na superfície da água. A abordagem usou filtro para detecção de bordas, descritor de textura, coleção de informações e dados ambientais processados por redes neurais. Foram feitos processamentos em imagens que apresentavam diferentes condições ambientais. Nesse estudo foi encontrada como valor ideal de velocidade do vento para a identificação das manchas de óleo o intervalo de 3,5 a 7,0 $\mathrm{m} / \mathrm{s}$ e o melhor ângulo de incidência para detecção na banda C é de $22^{\circ}$ a $40^{\circ}$.

Visando compartilhar dados oriundos de pesquisas sobre o uso de imagens SAR para a detecção de manchas de óleo no mar, Fustes et al. (2014) apresentam uma ferramenta, denominada Sentinazos, criada para detecção de vazamentos de óleo em áreas costeiras, cujo processamento de dados acontece na "nuvem", onde é possível a participação de usuários externos para o compartilhamento de informações e métodos dentro de uma estrutura integrada. Essa ferramenta permite que as imagens sejam pré-processadas, com calibração, filtragem e aplicação de projeção cartográfica. Para 
o processamento, foram incluídos na ferramenta métodos de detecção de manchas escuras, onde é feita a segmentação da imagem usando algoritmos específicos visando separar as manchas escuras que são de fato vazamentos de óleo, daquelas que são formadas por fenômenos naturais (falsopositivos). A plataforma permite que usuários externos incluam algoritmos na plataforma, que podem ser validados usando o grupo de imagens e respectivas máscaras já existentes. No trabalho, os autores apresentam ainda o resultado dos algoritmos que foram testados na plataforma e obtiveram melhores resultados. Sendo que dentre eles, o SKFCM (Spatially Constrained Kernelized Fuzzy C Means) obteve os melhores resultados com $86 \%$ de verdadeiros positivos e $98 \%$ de verdadeiros negativos.

No trabalho de Yang et al. (2016) foram feitas simulações numéricas em imagens SAR contendo vazamentos de óleo com o objetivo de estudar o mecanismo de espalhamento do óleo na superfície marítima, foram realizados testes considerando diversas condições de mar, de polarização do radar, de frequência e dos ângulos de incidência. O estudo verificou que as condições ideais consistem em alta frequência de radar e polarização VV das imagens.

Topouzelis et al. (2007) apresentam metodologia utilizando duas redes neurais, uma para a detecção das manchas escuras e outra para a classificação dos vazamentos e dos falso-positivos, usando imagens SAR de alta resolução. O método mostrou ser eficiente, sendo que a primeira etapa obteve acurácia de $94 \%$ das manchas escuras identificas e a segunda contou com a classificação correta de $89 \%$ das manchas. Para a segunda parte da detecção foram usados dados para discriminar os vazamentos de óleo dos falso-positivos considerando: relação do perímetro pela área (P/A), complexidade do objeto quanto à geometria, forma, desvio padrão (considerando os valores dos pixels).

Marghany (2001) desenvolveu um modelo para a detecção automática de óleo, que inclui análise da textura (para discriminar óleo da água) e os algoritmos Lee (para determinar a linearidade do movimento do óleo) e Gamma (para determinar o espalhamento do óleo). Para a análise da textura, foi calculada uma medida de textura especificada para cada pixel usando matrizes de co-ocorrência de nível de cinza, sem a realização de nenhuma filtragem anterior. Segunda o autor, a aplicação do método, especificamente em uma imagem RADARSAT de um acidente conhecido, permitiu distinguir a mancha de óleo da água de maneira adequada.

No trabalho realizado por Singha et al. (2013) foram usadas 38 imagens (ENVISAT ASAR e RADARSAT) visando treinar um sistema de detecção automático de óleo, contendo vazamentos de origem identificada e diversos falso-positivos. $\mathrm{Na}$ etapa de pré-processamento foi feita filtragem, retirada de ruídos e aplicação de máscara nas áreas de terra. A imagem pré-processada foi então segmentada para identificar as feições que poderiam ser vazamentos ou falso-positivos. Em seguida foi feita a seleção dos atributos com as características consideradas relevantes para a distinção das manchas, considerando elementos de retroespalhamento, forma, tamanho e textura. Na etapa seguinte foram usados dois classificadores, o primeiro constituído por árvore de decisão e o segundo por algoritmo de lógica difusa. Por fim, a metodologia criada foi testada em um conjunto de 118 imagens e a acurácia foi calculada, obtendo para as imagens ENVISAT 85,43\% de acurácia e $93,08 \%$ para as imagens RADARSAT.

Shi et al. (2008), seguindo a mesma sequência lógica, estabeleceram metodologia, usando mais de 600 imagens ERS-2 e Envisat SAR do norte do mar chinês, onde existiam 120 manchas de óleo conhecidas. Foi feito procedimento similar aos anteriores: pré-processamento da imagem, incluindo reamostragem, filtragem de ruído (filtro Lee) e correção do ângulo de incidência; segmentação de imagens e realce de manchas escuras; por último, a discriminação entre vazamentos de óleo e falsopositivos realizada manualmente por especialista, com base na ocorrência, tamanho, textura (forma, área, tipo de borda e homogeneidade) e contexto da mancha (considerando vento, correntes, localização e distância de pontos brilhantes nas imagens que costumam ser barcos e plataformas). Os resultados do processamento das imagens foram inseridos em um SIG com vetores com informações sobre: localização de plataformas, rotas de navios, linhas de costa e batimetria. Os dados geraram um mapa com a identificação de áreas de risco para poluição por óleo da área de estudo.

O trabalho de Guo e Zhang (2014) parte do princípio de que cada mancha escura identificada numa imagem de satélite, que tenha origens distintas, é constituída de diferentes materiais, tendo, dessa maneira, propriedades físicas diferentes e, com isso, adquirem diferentes formas. Assim os autores desenvolveram metodologia baseada em algoritmo que calcula as interações e relações de atributos

$\begin{array}{lllll}\text { Caminhos de Geografia } & \text { Uberlândia-MG } & \text { v. 21, n. 75 } & \text { Jun/2020 } & \text { p. 214-224 Página } 220\end{array}$


relacionados à forma da mancha. Foram usadas duas imagens ERS-2, onde as manchas escuras já estavam previamente identificadas como sendo vazamentos de óleo ou falso-positivos, a partir delas foram feitos os testes, visando verificar quais as relações de atributos representariam maior acurácia e quais não agregariam valor ao cálculo. Por fim, foi realizada classificação por aprendizado de máquina e por rede neural, sendo essa última a que apresentou melhor resultado. Os autores enfatizam a necessidade de durante o processo de detecção de vazamentos de óleo ser dado destaque para a seleção das características a serem usadas para a distinção entre manchas de óleo e falso-positivos, considerando que essa etapa é fundamental para a acurácia final do trabalho.

Lang et al. (2017) desenvolveram metodologia com o diferencial presente na fase de segmentação das manchas escuras, pois para eles essa etapa é crítica e fundamental uma vez que representa impacto significativo nas etapas seguintes. Inicialmente é feita calibração radiométrica, correção geométrica e remoção de speckle e ruídos; em seguida foi feita extração de características das manchas (níveis de cinza, geometria e textura); e por fim, foi realizada segmentação usando redes neurais artificiais e processamento morfológico. O resultado do experimento indica que a proposta de trabalho supera a performance de outras abordagens, tanto na acurácia da segmentação quanto na classificação das manchas encontradas.

Alpers et al. (2017) elencam desafios e equívocos encontrados em estudos realizados para identificação de óleo no mar, destaca-se nesse trabalho a apresentação de experiências para validação em campo de manchas encontradas em imagens de radar que apresentaram baixa performance, como o teste ocorrido no Mar Norte e Mar Báltico onde foram feitos testes com aeronaves equipadas com radar que identificaram 5 manchas de óleo, sendo somente 2 (duas) confirmadas após a coleta de amostra no local. Outro exemplo diz respeito à análise dos dados do banco CleanSeaNet, com imagens de diversas fontes europeias, foi constatada que somente 195 casos de $775(26 \%)$ foram confirmadas.

\section{DESAFIOS PARA A AUTOMAÇÃO}

Apesar de ter aumentando nas últimas duas décadas o número de pesquisas realizadas para a detecção de óleo no mar, a metodologia científica usada manteve-se constante, não ocorrendo mudanças das regras de detecção, sendo que o principal fator de mudança diz respeito ao fato de ter aumentado significativamente o número de imagens de radar disponíveis, que se tornaram mais acessíveis (TOPOUZELIS e SINGHA, 2016; FINGAS e BROWN, 2018).

Destaca-se a inviabilidade de realizar comparação entre as pesquisas e metodologias existentes, uma vez que elas fazem uso de variado conjunto de dados, relativos aos radares usados, aos recortes geográficos e às condições meteorológicas e oceanográficas de momento (TOPOUZELIS, 2007). Dessa forma, faz-se necessário a realização de testes com as distintas metodologias utilizando o mesmo conjunto de imagens pré-selecionadas que tenham manchas cuja origem seja conhecida, visando tornar possível a comparação quanto à acurácia dos métodos e, a partir dos resultados, propor novas formas de abordagem metodológica.

Segundo Zhang et al. (2014), apesar do aumento da acurácia e eficiência da detecção e classificação automática de vazamentos de óleo ocorrida em anos recentes, a probabilidade de eles operarem de maneira totalmente autônoma é pequena em virtude do número elevado de fenômenos imprevisíveis que podem ser observados em cada imagem SAR analisada, sendo necessário a participação de especialistas no processo.

Topouzelis e Singha (2016) destacam a necessidade de existir um banco de dados robusto contendo informações que tiveram verificação em campo tanto de vazamentos de óleo quanto de falsopositivos, em diversas regiões geográficas e com condições de mar distintas, além de informações sobre diferentes tipos de óleos.

Pesquisas recentes utilizando algoritmos de aprendizado profundo na etapa de classificação de imagens de satélite vêm apresentando resultados promissores que podem implicar no aumento da acurácia dos trabalhos desenvolvidos (CHENG et al., 2018). Esses algoritmos, quando comparados a outros comumente usados para a identificação de manchas de óleo na superfície marítima, demonstraram melhor performance (CHEN et al., 2017), podendo representar possibilidade de avanço na detecção automática de manchas de óleo em imagens de satélite. 


\section{CONSIDERAÇÕES FINAIS}

Este artigo apresentou o levantamento de pesquisas recentes relacionadas ao uso do sensoriamento remoto na detecção de vazamentos de óleo na superfície marinha, com destaque para os trabalhos que usaram imagens SAR, cuja predominância foi verificada, bem como o consenso quanto aos bons resultados no que diz respeito à distinção de manchas de óleo daquelas consideradas falso-positivos. Constatou-se que os mesmos passos metodológicos são usados no processamento das imagens (préprocessamento, segmentação das manchas escuras, estabelecimentos de critérios de decisão e classificação), havendo aperfeiçoamento em cada etapa. Nesse contexto, é necessário o desenvolvimento de novas pesquisas visando a realização de testes e criação de metodologias que permitam otimizar o tempo de análise das imagens, aumentar a eficiência na exclusão de falso-positivos e potencializar o nível de acerto das manchas escuras identificadas como vazamento de óleo. Para tanto, entende-se que o uso de algoritmos de aprendizado profundo representa grande potencial para aumento da acurácia dos resultados. Considerando o cenário brasileiro, onde a área de mar territorial é de grande dimensão e os recursos para vigilância são limitados, sobretudo a capacidade de ida a campo para a coleta de amostra de água, entende-se que o desafio maior seria o desenvolvimento de metodologia que não necessite de exemplos verificados em loco para a sua validação, principalmente quando considerado o número crescente de atividades relacionadas a exploração de petróleo e o número de vazamentos associados. Posteriormente, visando o dimensionamento do dano ambiental causado pelos vazamentos de óleo, devem ser pensadas pesquisas com objetivo de não apenas identificar a mancha, mas também identificar o tipo de óleo e calcular o volume derramado, considerando tamanho e espessura.

\section{AGRADECIMENTOS}

O presente trabalho foi realizado com o apoio da Coordenação de Pessoal de Nível Superior - Brasil (CAPES) - Código de Financiamento 001. Os autores agradecem também ao Conselho Nacional de Desenvolvimento Científico e Tecnológico (CNPq) e à Fundação de Apoio e Pesquisa do Distrito Federal (FAPDF).

\section{REFERÊNCIAS}

ABASCAL, A. J.; SANCHEZ, J.; CHIRI, H.; FERRER, M. I.; CÁRDENAS, M.; GALLEGO, A.; TURRELL, W. R. Operational oil spill trajectory modelling using HF radar currents: A northwest European continental shelf case study. Marine Pollution Bulletin, v. 119, n. 1, p. 336-350, 2017. https://doi.org/10.1016/.j.marpolbul.2017.04.010

ALPERS, W.; HOLT, B.; ZENG, K. Oil spill detection by imaging radars: Challenges and pitfalls.

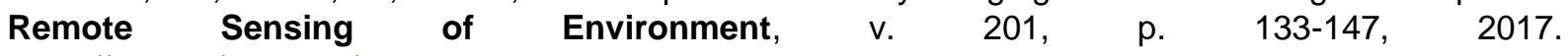
https://doi.org/10.1016/j.rse.2017.09.002

BENTZ, C. M.; POLITANO, A. T.; GENOVEZ, P. Monitoramento ambiental de áreas costeiras e oceânicas com múltiplos sensores orbitais. Revista Brasileira de Cartografia, v. 1, n. 57, 2005.

BRASIL, Lei no 9.966, de 28 de abril de 2000 (Lei do Óleo). Art. $2^{\circ}$ das definições e classificações. Disponível em: <http://www.planalto.gov.br/ccivil_03/LEIS/L9966.htm>. Acesso em: 29 outubro 2019.

BREKKE, C.; SOLBERG, A. H. Classifiers and confidence estimation for oil spill detection in ENVISAT ASAR images. IEEE Geoscience and Remote Sensing Letters, v. 5, n. 1, p. 65-69, 2008. https://doi.org/10.1109/LGRS.2007.907174

BREKKE, C.; SOLBERG, A. H. Feature extraction for oil spill detection based on SAR images. In: Scandinavian Conference on Image Analysis. Springer, Berlin, Heidelberg, p. 75-84, 2005. https://doi.org/10.1007/11499145 9

BREKKE, C.; SOLBERG, A. H. Oil spill detection by satellite remote sensing. Remote sensing of environment, v. 95, n. 1, p. 1-13, 2005. https://doi.org/10.1016/..rse.2004.11.015

CHEN, G.; LI, Y.; SUN, G.; ZHANG, Y. Application of deep networks to oil spill detection using polarimetric Synthetic Aperture Radar Images. Applied Sciences, v. 7, n. 10, p. 968, 2017. https://doi.org/10.3390/app7100968 
CHENG, G.; YANG, C.; YAO, X.; GUO, L.; HAN, H. When deep learning meets metric learning: Remote sensing image scene classification via learning discriminative CNNs. IEEE transactions on geoscience and remote sensing, v. 56, n. 5, p. 2811-2821, 2018. https://doi.org/10.1109/TGRS.2017.2783902

ESPEDAL, H. A.; JOHANNESSEN, O. M. Cover: Detection of oil spills near offshore installations using synthetic aperture radar (SAR). International Journal of Remote Sensing, v. 21, n.11, p. 2141-2144, 2000. https://doi.org/10.1080/01431160050029468

FINGAS, M.; BROWN, C. A review of oil spill remote sensing. Sensors, v. 18, n. 1, p. 91, 2018. https://doi.org/10.3390/s18010091

FINGAS, M.; BROWN, C. Review of oil spill remote sensing. Marine pollution bulletin, v. 83, n. 1, p. 9-23, 2014. https://doi.org/10.1016/j.marpolbul.2014.03.059

FISCELLA, B.; GIANCASPRO, A.; NIRCHIO F.; PAVESE, P. ; TRIVERO, P. Oil spill detection using marine SAR images. International Journal of Remote Sensing, v. 21, n. 18, p. 3561-3566, 2000. https://doi.org/10.1080/014311600750037589

FUSTES, D.; CANTORNA, D.; DAFONTE, C.; ARCAY, B.; IGLESIAS, A.; MANTEIGA, M. A cloudintegrated web platform for marine monitoring using GIS and remote sensing. Application to oil spill detection through SAR images. Future Generation Computer Systems, v. 34, p. 155-160, 2014. https://doi.org/10.1016/.j.future.2013.09.020

GARCIA-PINEDA, O.; HOLMES, J.; RISSING, M.; JONES, R.; WOBUS, C.; SVEJKOVSKY J.; HESS, M. Detection of Oil near Shorelines during the Deepwater Horizon Oil Spill Using Synthetic Aperture Radar (SAR). Remote Sensing. v. 9, n. 6, p. 567, 2017. https://doi.org/10.3390/rs9060567

GARCIA-PINEDA, O.; MACDONALD, I.; HU, C.; SVEJKOVSKY, J.; HESS, M.; DUKHOVSKOY, D.; MOREY, S. L. Detection of floating oil anomalies from the Deepwater Horizon oil spill with synthetic aperture radar. Oceanography. v. 26, n. 2, p. 124-137, 2013. https://doi.org/10.5670/oceanog.2013.38

GARCIA-PINEDA, O.; ZIMMER, B.; HOWARD, M.; PICHEL, W.; LI, X; MACDONALD, I. R. Using SAR images to delineate ocean oil slicks with a texture-classifying neural network algorithm (TCNNA). Canadian Journal of Remote Sensing, v. 35, n. 5, p. 411-421, 2009. https://doi.org/10.5589/m09$\underline{035}$

GUO, Y.; ZHANG, H. Z. Oil spill detection using synthetic aperture radar images and feature selection in shape space. International Journal of Applied Earth Observation and Geoinformation, v. 30, p. 146-157, 2014. https://doi.org/10.1016/j.jag.2014.01.011

JONES, B. A comparison of visual observations of surface oil with Synthetic Aperture Radar imagery of the Sea Empress oil spill. International Journal of Remote Sensing, v. 22, n. 9, p. 1619-1638, 2001. https://doi.org/10.1080/01431160010008564

KERAMITSOGLOU, I.; CARTALIS, C.; KIRANOUDIS, C. T. Automatic identification of oil spills on satellite images. Environmental modelling \& software, v. 21, n. 5, p. 640-652, 2006. https://doi.org/10.1016/i.envsoft.2004.11.010

LANG, H.; ZHANG, X.; XI, Y.; ZHANG, X.; LI, W. Dark-spot segmentation for oil spill detection based on multifeature fusion classification in single-pol synthetic aperture radar imagery. Journal of Applied Remote Sensing, v. 11, n. 1, p. 015006, 2017. https://doi.org/10.1117/1.JRS.11.015006

MARGHANY, M. RADARSAT automatic algorithms for detecting coastal oil spill pollution. International Journal of Applied Earth Observation and Geoinformation, v. 3, n. 2, p. 191-196, 2001. https://doi.org/10.1016/S0303-2434(01)85011-X

MISRA, A.; BALAJI, R. Simple approaches to oil spill detection using sentinel application platform (SNAP) - ocean application tools and texture analysis: a comparative study. Journal of the Indian Society of Remote Sensing, v. 45, n. 6, p. 1065-1075, 2017. https://doi.org/10.1007/s12524-016$\underline{0658-2}$

PISANO, A.; DE DOMINICIS, M.; BIAMINO, W.; BIGNAMI, F.; GHERARDI, S.; COLAO, F.; COPPINI, G.; MARULLO, S.; SPROVIERI, M.; TRIVERO, P.; ZAMBIANCHI, E. An oceanographic survey for oil spill monitoring and model forecasting validation using remote sensing and in situ data in the 
Mediterranean Sea. Deep Sea Research Part II: Topical Studies in Oceanography, v. 133, p. 132145, 2016. https://doi.org/10.1016/j.dsr2.2016.02.013

SHI, L.; IVANOV, A. Y.; HE, M.; ZHAO, C. Oil spill mapping in the western part of the East China Sea using synthetic aperture radar imagery. International Journal of Remote Sensing, v. 29, n. 21, p. 6315-6329, 2008. https://doi.org/10.1080/01431160802175447

SINGHA, S.; VESPE, M.; TRIESCHMANN, O. Automatic Synthetic Aperture Radar based oil spill detection and performance estimation via a semi-automatic operational service benchmark. Marine pollution bulletin, v. 73, n. 1, p. 199-209, 2013. https://doi.org/10.1016/. marpolbul.2013.05.022

TOPOUZELIS, K. Oil spill detection by SAR images: dark formation detection, feature extraction and classification algorithms. Sensors, v. 8, n. 10, p. 6642-6659, 2008. https://doi.org/10.3390/s8106642

TOPOUZELIS, K.; KARATHANASSI, V.; PAVLAKIS, P.; ROKOS, D. Detection and discrimination between oil spills and look-alike phenomena through neural networks. ISPRS Journal of Photogrammetry and Remote Sensing, v. 62, n. 4, p. 264-270, 2007. https://doi.org/10.1016/j.isprsiprs.2007.05.003

TOPOUZELIS, K.; SINGHA, S. Oil spill detection: Past and future trends. In: Proceedings of the ESA Living Planet Symposium. 2016.

YANG, W.; QI, C.H.; ZHAO, Z. Q. Numerical Simulation of Synthetic Aperture Radar Images for Ocean Oil Spill Pollution. Electromagnetics, v. 36, n. 1, p. 32-43, 2016. https://doi.org/10.1080/02726343.2016.1115461

YU, F.; SUN, W.; LI, J.; ZHAO, Y.; ZHANG, Y.; CHEN, G. An improved Otsu method for oil spill detection from SAR images. Oceanologia, v. 59, n. 3, p. 311-317, 2017. https://doi.org/10.1016/i.oceano.2017.03.005

ZHANG, Y.; LI, Y.; LIN, H. Oil-spill pollution remote sensing by synthetic aperture radar. In: Advanced Geoscience Remote Sensing. p. 27-50, 2014. https://doi.org/10.5772/57477

Recebido em: 30/10/2019

Aceito para publicação em: 26/02/2020 